\title{
IMPORTANCIA DE LAS DIFERENCIAS ENTRE ACTIVIDAD-PROCEDIMIENTO-PROCESO PARA LA AUTOEVALUACIÓN INSTITUCIONAL UNIVERSITARIA
}

\author{
Hernando A. Romero
}

\author{
Recibido 3-IX-1999 • Aceptado 14-IX-1999
}

\begin{abstract}
Resumen: Se refiere en una amplia reflexión, a que tener clara la diferencia de significados entre actividad, procedimiento-proceso, actividad social, proceso social y personal es muy importante en el campo de la autoevaluación institucional porque si no se reconocen sus diferencias, no se puede aludir correctamente lo que se entiende por actividad académica y las diferencias en el proceso pedagógico.
\end{abstract}

En el debate pedagógico nacional se viene dando un uso generalizado de la categoría proceso al lado del concepto actividad y la palabra procedimiento. Es más existe un uso indiscriminado de esas palabras confundiendo la una con las otras, como lo hace el CNA (1998, 1, 93-122) cuando sostiene que uno de los factores para la autoevaluación de los Programas Académicos es el de los "Procesos Académicos" y cuando hablan del factor organización, administración y gestión; en donde ubican al proceso organizacional al nivel de la actividad administrativa.

¿Por qué es importante tener claro esa diferencia de significados? Porque sin ella no se puede entender a la educación como un proceso personal, social e institucional que le da la naturaleza a las actividades sociales en que se expresan como parte de la sociedad y sus diferencias con la pedagogía. Pero más importante es el servicio que presta para la autoevaluación institucional y la prospección de sus modificaciones. Quien se queda en conocer la actividad sólo podrá generarle modificaciones parciales o cambios, situación que se da intensificando una de las manifestaciones de los componentes estructurales. Mientras que quienes pueden conocer los procesos podrán conocer la naturaleza del centro educativo, elaborar y realizar modificaciones totales, construyendo otra actividad educativa, con otra calidad. 
Por eso es que se considera una exigencia el que se entre a explicar qué se entiende por ACTIVIDAD, por PROCEDIMIENTO-PROCESO. ACTIVIDAD SOCIAL y por PROCESO SOCIAL Y PERSONAL.

La ACTIVIDAD es el conjunto de actos o eventos encaminados a un propósito $\mathrm{u}$ objetivo que son desarrollados por seres humanos. Son secuenciales, reiterativos y concatenados o encadenados por guardar una regularidad en su existencia; es decir, continuidad y permanencia en el tiempo y el espacio.

La actividad puede perder el motivo que la haya suscitado y convertirse en un acto aislado que transcurre como una secuencia de acciones que tal vez concrete una relación totalmente diferente con el mundo, posibilitando que surja otra actividad. Los actos pueden así adquirir una fuerza impulsora propia y llegar a convertirse en una actividad particular; por último los actos pueden transformarse para alcanzar un objetivo, en una operación capaz de efectuar diversas acciones.

Con esos actos los seres humanos actualizan sus necesidades tanto materiales como espirituales y resuelven sus conflictos. Los desarrollan a partir de unas motivaciones e intenciones en el plano personal o por una direccionalidad (propósitos) en el ámbito de grupos, colectivos o toda la sociedad. Se repiten constantemente como parte de la sociedad ya sea en el plano individual (actividad humano) o con otros seres humanos (actividad social).

La actividad implica una forma de relacionarse las personas con la naturaleza, la sociedad y consigo mismo. Son satisfactores en sí misma o medios para conseguir recursos para vivenciar otras necesidades.

Desde esta perspectiva los seres humanos son por las actividades que realizan. Aunque no se descarta en ellos la intencionalidad y la direccionalidad, reconociendo que lo determinante es lo que hacen, sin desconocer los aspectos subjetivos que intervienen en el acto que son diferentes a lo que creen que hacen. En el reconocimiento de su ser hay que hacer primar lo que hacen a la par de la intención y los motivos. De aquí que se reconozca que en toda actividad confluyan dos episodios: Unos objetivos (lo que acontece) y otros subjetivos (motivos, intenciones, intereses) que puede estar inspirada o no en una direccionalidad.

La intencionalidad se da en los seres humanos y la direccionalidad en los establecimientos o en toda la sociedad. La intencionalidad se da porque la actividad está inspirada en determinados objetivos, o por estar inspirada en valores, según la importancia que se le atribuya a determinados comportamientos, sin relación alguna con el resultado, o en estadios afectivos predominantes en el momento en el que el sujeto actúa y en la tradición por las costumbres arraigadas (Weber, 1974, 2, 20). La intencionalidad se da como la existencia de motivos en los sujetos.

Las actividades, según los momentos en su realización y el plano en el que se desenvuelve en el ser humano, pueden ser internas y externas. Las internas se van formando con el proceso de interiorización. Entendiéndose por interiorización "la transición de la que resulta que actos externos por su forma, con objetos también externos, materiales, se transforman en acciones que transcurren en el plano mental, en el plano de la conciencia a la vez son sometidos a una transformación específica, es decir, se generalizan, verbalizan, reducen, y lo principal es que se tornan capaces de continuar un desarrollo que transciende las posibilidades de la actividad exterior... De aquí que se plantee que la actividad exterior y la interna tienen una misma estructura común... Por consiguiente la actividad que es interna por su forma y deriva de la actividad práctica externa, no difiere de ésta ni se superpone a ella, sino que conserva un nexo de principio y además bilateral con ella (Leontiev, 1978, 3, 76-81). 
Con la interiorización se va formando la actividad interna entendida como ORIENTADORA por Galperin (1979, 4, 57-93). En otras palabras en sus relaciones con el mundo externo que lo rodea, o que incluye prioritariamente sus actividades externas, la actividad orientadora se da como internalización. Dicho en otras palabras, como reconstrucción teórica o mental de la operación de la actividad externa (Vygostki, 1979 ,5, 93) y de ella en su conjunto. Esa reconstrucción mental de los objetos se da como representación o metarepresentación, a escala racional, como imaginación y transracional. Esta se hace desde enfoques o cosmovisiones que el sujeto ha ido asimilando espontánea o conscientemente. Con la representación retoma las propiedades y con la develación de sus aspectos lógicos fundamentales, a partir de las relaciones que establece entre ellos, elabora la metarepresentación.

La representación es una IMAGEN cuyo contenido implica no sólo descubrir cosas aisladas y sus propiedades sino sistemas de objetos como totalidades, o sea, formada por elementos con relaciones mutuas.

Esas totalidades incluyen el sujeto mismo y sus autorrepresentaciones o metarrepresentaciones, los cuales puede usarse o no como bases para la ORIENTACIÓN en el mundo externo. La imagen, además, permite crear suposiciones sobre acciones posibles para resolver el conflicto que impide la satisfacción de la necesidad. Para eso, deben reconocerse sus resultados, los procedimientos posibles y recursos a usar a ser tenidos en cuenta, para después ser aprobados o rechazados con o sin modificaciones.

En las acciones ideales en el plano mental, se señalan las tareas en un orden secuencial, tales como:

a) una aclaración de la situación conflictiva existente,

b) una diferenciación del objeto que constituye la necesidad actual, c) la elección del camino, los recursos y de las formas de actuar,

d) la regulación de la actividad durante el proceso de ejecución.

También pueden darse actividades estereotipadas que se realizan involuntariamente. Mientras que la actividad orientadora consciente demanda de unas acciones voluntarias. Esta supone la conciencia del objetivo o del fin y la previa idea del procedimiento que puede conducir o realizarlo. Las acciones voluntarias son la capacidad y la fuerza espiritual del sujeto para realizar actos conscientes dirigidos con un propósito determinado y relacionados con el esfuerzo que se requiere y se realiza para vencer obstáculos que se presentan en el camino (Petrovski, 1986, 6, 361).

Después de la actividad interna, es decir de registrar la excitación, realización de la imagen y elaboración de la acción posible es cuando el ser humano, consciente, espontánea o estereotipadamente desarrolla la ACTIVIDAD EXTERNA OBJETAL.

Esta actividad orientadora está determinada internamente, por el nivel de desarrollo de los procesos de la naturaleza humana en el sujeto, en particular por el predominio entre ellos de los procesos cognitivos, o los emotivos, sentimientos superiores, etc. Pero también, por las posibilidades de interaccionar con otros durante su existencia y en términos generales, la sobredetermina la situación y posición social que el sujeto tenga en la sociedad dentro de la confrontación dominación/subordinación y por la influencia de la consciencia social y los saberes.

Mucha gente confunde la categoría de proceso con el significado de la palabra PROCEDIMIENTO; por éste se entiende la secuencia de pasos para realizar un evento, acto o hecho social. Son los momentos que se prevén y ejecutan generando unos acontecimientos que se manifiestan como episodios objetivos y subjetivos. Estos son acciones seguidas una tras otra con la que se 
configura un hecho social; guardan un orden con el anterior posibilitando a los subsiguientes.

Desde nuestra perspectiva la categoría de PROCESO no se entiende como la concibe el modelo lógico o enfoque sistémico, éste considera al Proceso como la ejecución de un conjunto de acciones, coordinadas al interior de una organización. Con ellas se instrumentalizan una serie de insumos para obtener un producto con unas propiedades o características externas específicas a las que se les exige un máximo de excelencia.

Según ese enfoque el proceso es algo que no tiene principio ni fin pues se "realimenta" a si mismo con un control permanente y en una relación de causalidad circular y no lineal ni multidimensional. Se presenta como la introducción de unos insumos, sacados de un contexto para ser instrumentalizados con el objetivo de obtener un producto cuyas características deben ser permanentemente diseñadas con anticipación y con un cálculo preciso y controladas para que se den según lo establecido previamente.

Este enfoque sistémico surgió por la racionalización, justificación y exposición sistemática del FUNCIONAMIENTO de los procesos productivos fabriles o industriales que fueron utilizados para explicar tanto los fenómenos naturales como los procesos sociales.

Dicho enfoque al ser aplicado al conjunto de la sociedad inició la época de la concepción de la razón conocida como la "razón instrumental". Está en el campo de la educación, al ser aplicada, hizo posible la creación de la teoría pedagógica del Diseño Instruccional que inspira el conjunto del Modelo Educativo Tecnológico Curricular.

Este Modelo concibe el proceso educativo como la confluencia de unas informaciones, sacadas del contexto, para que, a través de unas acciones ejecutadas por unos agentes instructores orienten a otros sujetos-objetos (estudiantes) en la instrumentalización de la información para que se expresen, en ellos como unas conductas terminales alcanzadas como objetivos específicos observables, al concluir cada evento pedagógico. E incluso, puedan ser medidas a través de unos instrumentos "fiables"; los test, o pruebas "objetivas", etc.

Para el modelo lógico holísta o integrador el PROCESO es la forma de existencia del DESARROLLO de los fenómenos del mundo. Es una palabra que procede del latín "procesus" y que quiere decir marcha adelante $o$ ir adelante. Aunque como categoría el proceso no sólo conlleva el ir hacia delante, sino también puede ser el retornar o el involucionar. El proceso se explica develando la génesis, el devenir o secuencia de su existencia desde cuando fue creado o inventado hasta el momento en que se pretende explicar el fenómeno objeto de la investigación, reconoce además su estructura y las cualidades o la naturaleza de sus elementos y su funcionamiento.

Es ante todo la capacidad interna de automovimiento de un fenómeno, de autodinamizarse en su devenir. Es la forma de existencia del ser de estar siendo y dejando de ser. El hombre cuando aborda un campo de investigación no lo hace con el ser del mismo sino que se enfrenta al estar siendo y el dejando de ser del mismo por cuanto su existencia se da como un eterno modificarse.

El proceso expresa la secuencia que marca la dinámica de las fuerzas internas que autodinamizan o generan el movimiento de un fenómeno; fuerzas éstas que a su vez le dan su naturaleza. Este movimiento es específico, dinámico y autocentrado y no mecánico. Llega hasta la negación del fenómeno mismo; esta negación puede darse como una reproducción-repetición-conservación-crecimiento o como una destrucción-reconstrucción. La negación genera, a su interior y exterior, pequeñas pero sucesivas modificaciones parciales progresivas o regresivas para su mejoramiento o envilecimiento o pronta extinción o para su total transformación. 
Esto quiere decir que todo fenómeno tiene su génesis: ya sea de la sociedad, los saberes sociales, los sentimientos; además, tienen un origen si se trata de fenómenos naturales y a partir de ellos un devenir. Se entiende por génesis el momento de la creación del acto, el hecho social o del proceso social en el tiempo y el espacio. Es el momento de su insurgencia como un acto o algo diferente en la historia de la sociedad.

Este fenómeno no permanece igual ni en el tiempo ni en el espacio por el encadenamiento de fuerzas internas que rigen su devenir y lo determinan. Es una autodinámica que no le deja permanecer en el momento inicial cuando surgió; es impelida por fuerzas internas que proceden del ser mismo. "En los seres vivos se conoce esa dinámica como autodinamismo. Mientras en los seres humanos como cualidades generadas por su constante interaccionar con los demás, la naturaleza y consigo mismo y al realizar la internalización. En el sujeto se da estructurando el mundo y estructurando a su vez sus propios instrumentos de estructuración" (Piaget, 1987, 7, 118-119).

Por devenir se entiende, además, a los diversos períodos secuenciales en la existencia del fenómeno. Con la precisión del significado del devenir es posible entender las secuencias en que transcurre la investigación de un hecho, ¿Qué diferencias establecer durante el fluir del proceso cuando se aborda para su reconstrucción teórica? Durante el fluir de un proceso surgen las fases, las etapas, el estadio y el estado, lo que conlleva a su obligatoria diferenciación. Este devenir está determinado por la ESENCIA de los hechos, no es más que la correlación de fuerzas de factores internos al hecho o al proceso social que son contradictorios y le dan la naturaleza específica, diferenciándolos de otros históricamente determinados. Aquí el desarrollo está dado por la "lucha" o unidad de los contrarios mutuamente excluyente y en relaciones recíprocas. El uno no pue- de existir sin el otro. Es la fuente del movimiento progresivo o regresivo.

Las reacomodaciones de las relaciones de fuerzas entre los factores internos y las exigencias extemas se dan precisando una secuencia. Éstas transcurren durante lapsos de corta, mediana o más o menos larga duración. Según ésta pueden conformar períodos o subperíodos que se denominan: fases y dentro de éstas se dan las etapas. Ellas tipifican formas específicas de expresión de las relaciones de fuerzas estrucuturales tanto internas como con las del contexto. Un ejemplo es el de los seres humanos: no nacen adultos. Desde el nacimiento transcurren por fases y dentro de éstas por etapas: (Merani,1968, 8, 77-78).

Cuando se aborda una etapa para establecer en un momento su situación o coyuntura, o sea para establecer cómo está la correlación de fuerzas entre los factores determinantes de la existencia del fenómeno, el nivel de los progresos o avances o retrocesos de cada una de las relaciones, o sea cómo están reacomodadas o reorganizadas, se puede hablar de que el fenómeno se está abordando en su ESTADIO.

El estadio expresa las fronteras del desarrollo en un momento. Son fronteras de crecimiento pero fundamentalmente de su nivel o grado de cualificación global e históricamente comparada y determinada. En el caso de los niños puede ser el momento del abordaje de su conocimiento al llegar al aula. Es el momento que se denomina: "estadio de desarrollo" pues se da una especie de "corte vertical” para ver cómo ha evolucionado y en cual se expresa su desarrollo a partir de los diversos procesos de su naturaleza humana.

Se concibe por ESTADO del fenómeno a las dinámicas globales que confluyen cuando la correlación de fuerzas favorece la reconstrucción, reproducción, o sea la conservación, consolidación y mantenimiento del hecho y del proceso que generan, e incluso si garantiza su crecimiento en ascenso y relativamente permanente, o 
favorecen la destrucción reconstrucción expresando profundos desequilibrios en el fenómeno. Estos criterios hay que tenerlos en cuenta cuando se aborde la reconstrucción teórica de un sistema de fenómenos convirtiéndolos en campo de investigación.

El primer ESTADO particular se denomina de REPOSO, de "tranquilidad, "de armonía”. El REPOSO se da por el predominio del "estar siendo" del fenómeno o de un proceso educativo. Pero cuando la correlación de fuerzas comienza a variar para buscar nuevos reacomodos según las exigencias del contexto, para avanzar, retroceder o negar el fenómeno, se genera inestabilidad en ellos. Esta puede ser relativa o absoluta. Es relativa cuando es parcial por ser pequeñas crisis que jalonan la superación de una etapa a otra, o de una fase a otra. Aquí se da negación conservación, ampliación.

Pero es absoluta o total si lo que se pretende es la negación - destrucción - reconstrucción total para dar génesis o surgimiento a otros hechos diferentes a los que existen. Este ESTADO se reconoce como de CRISIS o MOVIMIENTO MANIFIESTO. Durante este ya no predomina el estar siendo sino el dejar de ser. Es el predominio no de la superación parcial sino total. Se da desequilibración en las prácticas sociales y viejos esquemas de acción, de pensar y sentir para darle paso a la reequilibración incrementante.

El concepto crisis denota el momento en el desarrollo de cualquier fenómeno durante el cual se da la posibilidad de la negación de lo existente y el surgimiento de su alternativa desde su seno. Es el momento de la desarticulación de las partes componentes o de los elementos que integran un sistema con una estructura específica y en la cual ésta se desarticula y por lo tanto las funciones de cada uno de esos elementos no permiten la realización de la operatividad esencial de ese fenómeno. Es el momento en que lo nuevo no tiene fuerza para aparecer y lo viejo no quiere o tiene suficiente fuerza aún para desaparecer. La crisis o bien posibilita a lo viejo o recomponerse para reorientar su desarrollo, si lo nuevo no tiene una correlación de fuerzas suficiente para hacer desaparecer al viejo fenómeno, conformarse como crisis cíclicas. O por el contrario, si la tiene es el momento en que se posibilita su transformación, es decir su ruptura.

Se puede concluir que a nivel de $E S$ $T A D O S$ se encuentran dos períodos diferentes, el de reposo y el de cambio manifiesto o desequilibración. En el primero predomina el estar siendo y en el segundo el dejar de ser para dar paso a un nuevo ser con otra calidad. En esta transición del uno al otro fenómeno o hecho social se da la reequilibración. Para el caso del desarrollo de los saberes sociales elaborados su estar siendo los convierte en ciencia oficial y su dejar de ser implica su superación por otro más complejo o multilateral en su explicación, valoración y planteamiento de innovaciones a la parcela del mundo, campo de investigación. Esa superación de la una por la otra es la revolución científica de la que habla Khun.

Para abordar el conocimiento de un centro educativo se parte de interactuar con los sujetos protagonistas de los hechos con los episodios de la vida cotidiana del mismo, y después se entra a establecer su funcionamiento, estructura, esencia para después buscar cómo se ha dado ésta en su desarrollo.

Cada hecho social tiene su operatividad: ésta se conoce como FUNCIONAMIENTO. Aparece en una reproducción circular de momentos tales como: la programación, organización, orientación, ejecución-control y evaluación.

Otro de los aspectos lógicos fundamentales, es el relacionado con la $E S$ TRUCTURA.

Ella se da por un conjunto de elementos o partes que establecen unas relaciones dinámicas y estables del hecho y de los procesos educativos. La estructura, a 
su vez está determinada, en su calidad, por la ESENCIA y tienen una forma de operar desde su inicio hasta su finalización relativa en el tiempo y en el espacio.

La esencia para los hechos o procesos educativos, representa su CALIDAD y está dada por los factores internos pero en una interdependencia con la naturaleza de los avances y naturaleza de la sociedad en donde fueron creados. Es en un flujo de relaciones para responder a esos avances de la sociedad y del período histórico lo que determina en última instancia su naturaleza.

Se da por cuanto el ser de los hechos o procesos educativos están en una permanente inclinación, proyección o dinámica que reorganiza sus fuerzas internas, o sea autorregula en una u otra manera su existencia. Cada intento o logro de una organización de las relaciones internas según las exigencias externas, implica una innovación parcial o total en la correlación de fuerzas de su totalidad y encausa de nuevo su dinámica de desarrollo.

Para finalizar lo referente al PROCESO se podría resumir su significado de la siguiente manera: es la capacidad de automovimiento de los fenómenos, de autotransformación que se dinamizan por la confluencia de múltiples factores internos y externos al mismo: es un estar siendo y dejar de ser. Un ser devenido y en devenir permanente. En el proceso se da o constituye la naturaleza ontológica a la actividad o de cualquier fenómeno. Es la forma de existencia del desarrollo de los fenómenos tanto materiales como espirituales. $A$ la actividad la determinan tanto procesos sociales como procesos personales.

Por eso se concibe al proceso social como los aspectos lógicos que determinan la confluencia reiterativa de actividades sociales y humanas de los sujetos o seres humanos de una comunidad, localidad, región o país. Están presentes y caracterizan la naturaleza de las actividades sociales y humanas específica que, en forma cohesionada y durante cierto período de tiempo, la cual realizan los seres humanos para vivir, actualizar o incrementar sus necesidades humanas y resolver conflictos colectivos.

En otras palabras por Proceso Social se entiende la unidad de múltiples determinaciones que dinamizan internamente el devenir de las actividades sociales; es la confluencia reiterativa de relaciones sociales que determinan las posibilidades de la actividad y que son similares en el tiempo y el espacio por cuanto no existen como tales en una forma ahistórica o aisladas de un conglomerado social determinado; es más se dan dentro de hechos sociales concretos. Los procesos sociales son los que determinan, como factores internos, a las actividades sociales; entre los procesos se encuentran como fundamentales las relaciones sociales de dominación/subordinación y o dominación/colaboración social, como se dijo antes.

Los Procesos personales se dan porque en los seres humanos a lo largo de su existencia manifiestan una dualidad de procesos que determinan tanto las actividades externas como las orientadoras o internas. En las externas inciden los procesos físicos, prácticos sociales (trabajo de ejecución y de dirección, además del trabajo manual e intelectual) y Procesos Socializantes (trabajo individual y de colaboración social: trabajo de grupo y de colectivos). Mientras que en las actividades internas influyen los procesos mentales cognitivos, emotivos de sentimientos superiores y de comunicación. Las internas no son más que resultado de la Internalización de las externas por ser expresión de la reconstrucción interna de las operaciones externas, al decir de Vygotski (1979, 5, 93) e incluso considera que las interacciones entre ambas actividades se conciben dentro del marco de las relaciones biológicas de la conducta y las condiciones sociales, a través de las cuales se lleva a cabo la actividad humana. 
Reconocer la importancia de las actividades es fundamental porque implica validar la tesis de que una sociedad y el ser humano manifiestan lo que son, solamente a partir de sus actividades, en las que están explícitas unas prácticas sociales. No se les puede juzgar por lo que piensan de sí mismo o sobre lo que realizan sino a partir de sus actividades sociales en sus dimensiones objetivas y subjetivas ubicándolas en las relaciones sociales históricamente determinadas, por cuanto cada ser humano a pesar de conservar su individualidad es un ser colectivo moldeado por la ideología dominante. Esto debido a que con estas generan procesos ya sean sociales, económicos, políticos o culturales. Es más sostienen y reproducen dichos procesos, pero también lo pueden involucionar o generar progresos al realizarle innovaciones parciales o totales.

\section{¿Qué implicaciones conlleva el tener la claridad de los significados del concepto actividad y la categoría proceso?}

En el campo de la Autoevaluación institucional porque si no se reconocen sus diferencias no se puede hacer alusión correctamente a lo que se entiende por ACTIVIDAD ACADÉMICA y las diferencias con el PROCESO PEDAGÓGICO y se puede caer en el error de hablar de PROCESO ACADÉMICO. Ellas hacen posible reconstruir teóricamente aspectos que son diferentes como el de fenómeno y esencia. La actividad es sinónima de fenómeno y el proceso a esencia. Este le da la naturaleza al fenómeno y aquella lo dinamiza mientras ella existe.

Hablar de procesos académicos es como hablar de un "ser vivo serpiente" o "vegetal árbol". Es lo que pasa cuando los del CNA $(1998,1)$ lo usan dándole como significado a aquel que hace referencia a las manifestaciones de un proceso pedagógico específico que son aspectos relacionados con la actividad académica: y es a ésta a la que diseña o prospecta cualquier proyecto académico, así sea el curricular; tal significado a la palabra proceso académico se lo dan cuando hablan de que "si el curriculo contribuye a una formación integral en los conocimientos, métodos y principios básicos de acción de la disciplina, profesión, ocupación u oficio respectivo y si es coherente con los objetivos del Programa"... "si promueve la formación integral del estudiante", "si es flexible, actualizado y pertinente"... "si las metodologías empleadas para el desarrollo de los contenidos del plan de estudio son coherentes". Y así se podrían relacionar todas las otras características de dicho factor; desde la 28 a la 43 del texto sobre "Lineamientos para la acreditación".

Cuando se hace referencia a la formación en los conocimientos desconoce que éstos son la expresión de un tipo de objeto de enseñanza: los teóricos que son un tipo de componente de todo proceso pedagógico. O cuando hablan de la formación integral que son a su vez, la manifestación fenoménica de la esencia del tipo de Desarrollo Humano por ayudar a formar, por exigencias del desarrollo progresivo de la Humanidad.

Si se sigue usando dicho concepto de procesos académicos no se podrán realizar las transformaciones en los Programas Académicos que la sociedad exige y que el solo conocimiento de las propiedades de los fenómenos no lo posibilitan, situación que si facilita el conocimiento del proceso. A no ser que se quiera imponer el Modelo Ideal como Tipo Ideal (Weberiano) u óptimo por construir que representaría el ideal de calidad de la educación que todo centro educativo debe reunir y que en este caso se presenta en forma vergonzante por cuanto no se reconoce como un Modelo Educativo tomado del saber popular universitario en el cual ha primado el Modelo Educativo Tecnológico Curricular. 
La actividad expresa el aspecto externo de los fenómenos mientras que el proceso representa los aspectos internos del mismo; sus aspectos lógicos fundamentales ya explicados (como lo son la esencia, la tendencia o génesis y devenir, la estructura y el funcionamiento). La actividad se conoce por la percepción posibilitando su descripción, mientras que el proceso requiere, para ser conocido de un proceso racional que se da por la interpretación (develar los problemas), la explicación y la valoración crítica. Un conocimiento que nos posibilite la transformación de cualquier fenómeno conlleva a que se conozcan las propiedades fenoménicas y la esencia, tendencia, estructura, funcionamiento o proceso. Este se manifiesta a través de aquel.

La "esencia es el aspecto interno, relativamente estable, de la realidad objetiva que permanece oculto tras la superficie de los fenómenos y que se manifiesta a través de ellos. Mientras que el fenómeno es el aspecto externo más movible y cambiante de la realidad objetiva, que constituye la forma de manifestarse la esencia. Toda esencia se manifiesta de una manera o de otra. Cada fenómeno presupone su propia esencia. Todos los fenómenos diversos, que no se asemejan entre si, tienen, sin embargo, una esencia común; la esencia expresa algo universal, en tanto que el fenómeno hace patente algo singular" (ROSENTAL, 1965, 9, 55).

Por eso se puede concluir afirmando que no siempre ha existido el tipo de actividad educativa vigente, pues ellas han sido históricamente diferentes conformando Modelos Educativos específicos.
El conocimiento de estos lo posibilita el tener otro significado para la palabra proceso.

\section{Referencias bibliográficas}

CNA. Lineamientos para la Acreditación. Santafé de Bogotá. CNA. 1998.

WEBER, Max Economía y Sociedad. México Fondo de Cultura Económica. 1974.

LEONTIEV, A.N. Actividad, Conciencia y Personalidad. Ediciones Ciencias del Hombre. Buenos Aires. 1978.

GALPERIN, P.Y. Introducción a la Psicología un enfoque dialéctico. Pablo del Río Editor Madrid. 1979.

VYGOTSKI, Lev. El desarrollo de los procesos psicológicos superiores. Barcelona: Grupo Editorial Grijalbo. 1979.

PETROVSKI. A. Psicología General: Manual didáctico para Institutos de Pedagogía. Editorial Progreso, Moscú. 1986.

Piaget, J. y García, Rolando. Psicogénesis e historia de las ciencias. México Editor Siglo XXI. 1987.

Merani, Alberto. Psicología Genética. México Editorial Juan J. Grijalbo. 1969.

Rosenta, M. y Straks, G. Categorías del Materialismo Dialéctico. México, D.F. Editorial Grijalbo, S.A. 1965. 